\title{
Effects of Small Conductance Calcium Activated Potassium Channels in Cardiac Myocytes
}

\author{
Angelina Peñaranda, Blas Echebarría, Enrique Alvarez-Lacalle, Inmaculada R. Cantalapiedra \\ Departament de Fisica, Universitat Politècnica de Catalunya, Barcelona, Spain
}

\begin{abstract}
Among the different potassium channels present in cardiac myocytes, the small conductance Ca2+ activated potassium channels (SK channels) are particular because they are affected by changes in intracellular calcium. The functional role of these channels in cardiac electrophysiology is still under intense debate. While they do not seem to play an important role in healthy heartstheir associated current, $I_{K C a}$, is smaller than other potassium currents -, there is increasing evidence that they may become relevant under pathological conditions. In fact, both pro- and anti-arrhythmic effects have been assigned to these channels, depending on the clinical situation. In this work, we have incorporated the current through SK channels, $I_{K C a}$, into an electrophysiological ionic model of human atria myocyte. This allows us to evaluate changes in the action potential under different parameters affecting the kinetics of these channels. We observe a large dependence of the action potential duration with the conductance and gate dynamics of the channel. The dependence of SK channels with changes in intracellular calcium dynamics helps decreasing the proarrhythmic effect of spontaneous calcium release events.
\end{abstract}

\section{Introduction}

Atrial fibrillation (AF) is the most common cardiac arrhythmia, with an incidence that increases with age. Although the development of AF can be multicausal, an often underlying factor is the occurrence of early or delayed depolarizations, caused by instances of spontaneous calcium release. In this, a sudden increase in intracellular calcium activates the sodium-calcium exchanger, depolarizing the cell membrane. The SK channels, which also open with calcium, may help to counteract this effect of the pump. This possible antiarrhythmic effect of these channels has opened the perspective of finding a new therapeutic target in the treatment of AF.
Still, there is contradictory evidence on the pro- or anti-arrhythmic role of these channels. The relation between SK expression and AF has been confirmed by a genomic study that reveals that polymorphisms in the calcium-dependent potassium-channel KCNN3 gene are an important risk factor for atrial fibrillation [1]. However, despite the relationship between dysfunction of these channels and AF it is not clear the character pro- or anti-arrhythmic of their inhibition.

In relation to their functionality, inhibition of SK channels by apamin has little effect on the action potential duration (APD) in normal ventricular myocytes but a prolongation of action potential (AP) is observed in atrial cells, providing evidence of the contribution of these channels in the action potential repolarization in atria. Transgenic mouse with overexpression of SK channels showed abbreviation of APD and refractory period as well as increasing susceptibility to arrhythmias and sudden death [2]. Opposed effects were found in genetic ablation of SK channels in mouse, originating prolongation of APD, increasing the incidence of early after depolarization (EAD) and induction of AF [3]. Channel inhibition in canine atria is linked to heterogeneous APD, dispersion in repolarization, occurrence of alternans and re-entrant arrhythmias [4]. The controversy in these results could correspond to different mechanisms underlying the arrhythmic processes.

In this paper we perform simulations of a model of human atrial myocyte in which the current through SK channels, $\mathrm{I}_{\mathrm{KCa}}$, is included. We analyse the effect of different parameters, with special emphasis on those corresponding to the dynamics of the SK channel gate. We consider the effect of both a constant and a calcium dependent gate activation time. Likewise we study the effect of $\mathrm{I}_{\mathrm{KCa}}$ in the case of occurrence of spontaneous calcium release from the sarcoplasmic reticulum able to produce delayed depolarizations. 

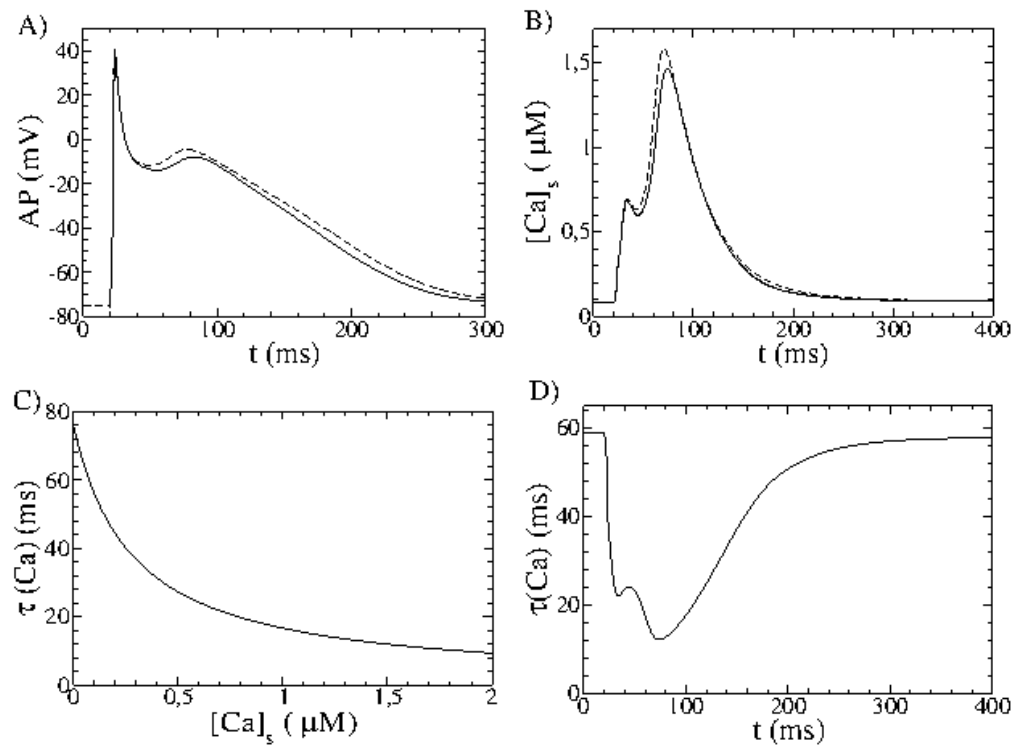

Figure 1. A) AP and B) subsarcolema calcium concentration, $[\mathrm{Ca}]_{\mathrm{s}}$, for a stimulation period of $T=1000 \mathrm{~ms}$. Solid lines correspond to calcium dependent constant time gate, $\tau(\mathrm{Ca})$, with values of half activation calcium concentration, $K_{\mathrm{KCa}}=700 \mathrm{nM}, q=2$ and conductance $g_{K C a}=0.005 \mathrm{~ns} / \mathrm{pF}$. Dashed lines represent the same magnitudes when current through SK channel is not included. C) Variation of calcium dependent time constant of the gate, $\tau(\mathrm{Ca})$, with the calcium concentration. D) Evolution of $\tau(\mathrm{Ca})$ for the AP showed in A).

\section{Material and methods}

Small conductance calcium activated potassium (SK) channels have been identified in different species and cell types. In the heart, their functionality has been studied in several species [5], expanding its study in the last decade. We investigate the effect of $\mathrm{I}_{\mathrm{KCa}}$ current on the atrial action potential introducing this current in the human atria model by Lugo et al. [6]. The current is modelled assuming an ohmic dependence on transmembrane voltage and an activation gate for the SK channels, whose steady state presents a sigmoidal dependence on intracellular calcium in the subsarcolemal space. The current can be written as [7]:

$$
I_{\mathrm{KCa}}=g_{K C a} f(\mathrm{Ca})\left(\mathrm{V}-V_{K}\right)
$$

being $g_{K C a}$ the conductance of the channel and $V_{K}$ the Nernst potential for the potassium ions. The gate $f(\mathrm{Ca})$ satisfies the equation:

$$
\frac{d f}{d t}=\frac{f_{\infty}-f}{\tau}
$$

with

$$
f_{\infty}=\frac{C a^{q}}{K_{K C a}^{q}+C a^{q}}
$$

The constant $K_{K C a}$ is the half activation calcium concentration, the Hill coefficient $q$ takes account of the cooperativity effect and $\tau$ is the activation time. In the literature there exist a disparity of values for the $I_{K C a}$ current parameters. We have studied the sensitivity of the results to different parameter values. Since, in a different work [7], we observed that the cooperativity coefficient $q$ does not affect much the action potential, we have set it to a fixed value $q=2$. As standard values we also set the conductivity $g_{\mathrm{KCa}} / C_{m}=0.005 \mathrm{nS} / \mathrm{pF}$ and $K_{\mathrm{KCa}}=700 \mathrm{nM}$. In this work we consider a calcium dependent gate activation time, given by the following expression [8] (with $\mathrm{Ca}$ in $\mathrm{M}$ )

$$
\tau(C a)=\frac{76}{3.572 C a+1}
$$

although, for comparison, some simulations are also conducted with constant values of $\tau=5 \mathrm{~ms}$ and $75 \mathrm{~ms}$.

Our aim is to analyse how changes in SK physiology may affect the AP as well as the APD in the Lugo model.

\section{Results}

In Figure 1 we present the effect of this current in the form of the AP and in the calcium concentration in the subsarcolemal space. The main effect occurs during repolarization. In the same figure we show the dependence of the gate activation time on calcium concentration and its change during an AP. The activation time is faster in the initial phases of the AP (down to $\tau$ $12 \mathrm{~ms}$ ), and slower during the repolarization phase. This behavior is reproduced in Figure 2, in which comparison with a faster constant value of $\tau=5 \mathrm{~ms}$ is shown. The opening probability of the faster gate practically follows the 

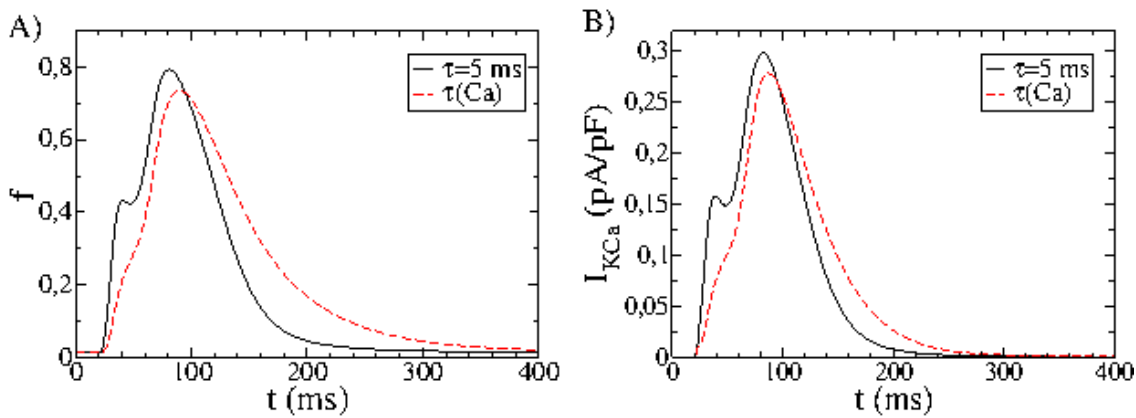

Figure 2. A) Comparison of the effect of faster activation constant time and calcium dependent constant time in the SK gate $f(C a)$, (A)) and $\left.I_{\mathrm{KCa}}(\mathrm{B})\right)$. The rest of parameters have the same value as in previous figure.

behaviour of calcium, while the $\tau(\mathrm{Ca})$ case softens this dependence, remaining opened for a longer time and thereby increasing the contribution of the $I_{\mathrm{KCa}}$ current in the repolarization phase.

The impact of the current on the AP is produced predominantly in the phases two and three of repolarization. A faster gate has a larger effect in phase two, but it is when the gate activation time is calcium dependent, $\tau(\mathrm{Ca})$, that the repolarization in phase three and four is more pronounced, with the consequent reduction in $\mathrm{APD}_{90}$ observed in restitution curves.

The variation of $\mathrm{APD}_{90}$ as a function of the SK conductance is shown in Figure 3, for several activation time constants. For a rate of $1 \mathrm{~Hz}$ there is a bimodal dependence of $\mathrm{APD}_{90}$ with conductance. In the case of a fast gate $(\tau=5 \mathrm{~ms})$, there is an initial increase of $\mathrm{APD}_{90}$ with conductance. This is not present for the slow gate, or for the calcium dependent time constant $\tau(\mathrm{Ca})$, where the increase appears for higher values of the conductance. The fast and calcium dependent $\tau(\mathrm{Ca})$ gates show a convergent behaviour at large conductances. For a stimulation rate of $2 \mathrm{~Hz}$, the initial decrease of $\mathrm{APD}_{90}$ is common for all gates kinetics, but evolves in different ways for each case. While the $\mathrm{APD}_{90}$ monotonously decreases for the slow gate, it again increases at higher conductance in the other two cases. Particularly interesting is the appearance of a bifurcation for the fast gate, i.e. $\tau=5 \mathrm{~ms}$, presenting alternans that could be related to phenomena associated with overexpression on SK channel.

The restitution curves are also represented in Figure 3. For rapid stimulation, both fast and calcium dependent gates present a similar behaviour, that becomes more divergent as the stimulation period increases. This effect is especially relevant for high conductance. Variation of $\mathrm{APD}_{90}$ due to the $I_{\mathrm{KCa}}$ current is insignificant at $T=1000 \mathrm{~ms}$ for the fast gate, but relevant for $\tau(\mathrm{Ca})$.

As has been shown elsewhere [7] the $I_{\mathrm{KCa}}$ current does not only affect the APD, but it can also be considered as a safety factor in front of spontaneous calcium release occurrences, reducing or avoiding their effects. To analyse this possibility we simulated situations in which the opening of ryanodine receptors (RyR) originated a spontaneous release. We compared similar situations with and without $\mathrm{I}_{\mathrm{KCa}}$ current. Results for two different opening probabilities and time of openings are shown in Figure 4 for a stimulation period of $T=1000 \mathrm{~ms}$. This shows that, beyond decreasing the depolarizing effect of spontaneous calcium release (left panel of Fig.4), the presence of the $I_{\mathrm{KCa}}$ current can avoid altogether the development of an extra depolarization (right panel, Fig.4). For the conditions and rhythm presented in the figure both constant and calcium dependent gate kinetics produce practically the same quantitatively effects.
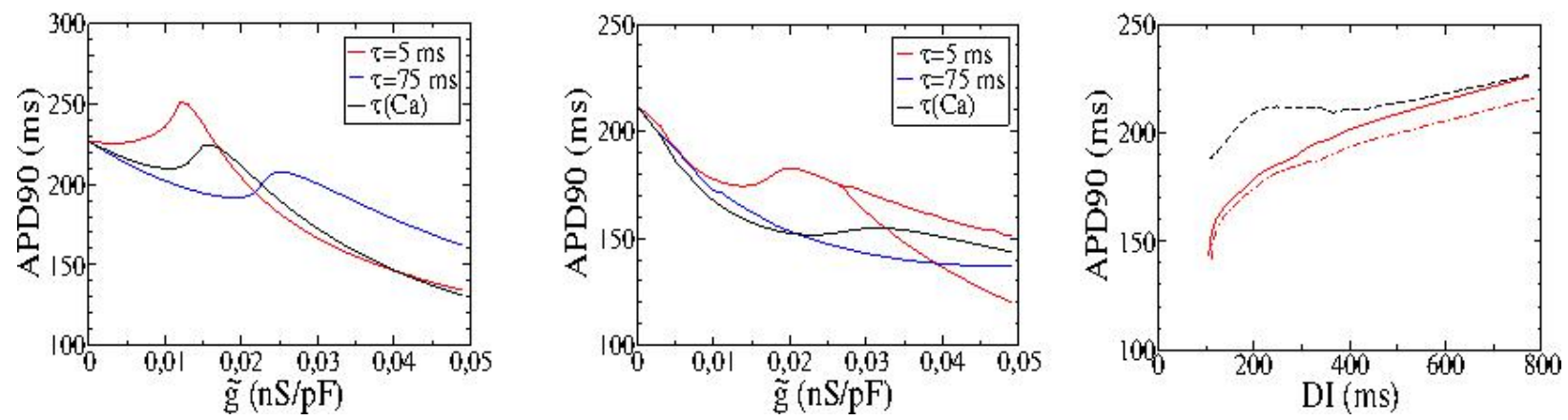

Figure 3. $\mathrm{APD}_{90}$ versus conductance of the SK channel for different activation time constants, corresponding to stimulation periods of $\mathrm{T}=1000 \mathrm{~ms}$ (left) and $500 \mathrm{~ms}$ (midle). $\tau(\mathrm{Ca})$ represents calcium dependent time constant of the gate. In both cases $q=2$ and $K_{\mathrm{KCa}}=$ $700 \mathrm{nM}$. On the right panel, restitution curves for conductance value $g=0.005 \mathrm{~ns} / \mathrm{pF}$, for $\tau=5 \mathrm{~ms}$ (red solid line) and $\tau(\mathrm{Ca})$ (red dashed line). Dashed black restitution curve corresponds to $I_{\mathrm{KCa}}=0$. 

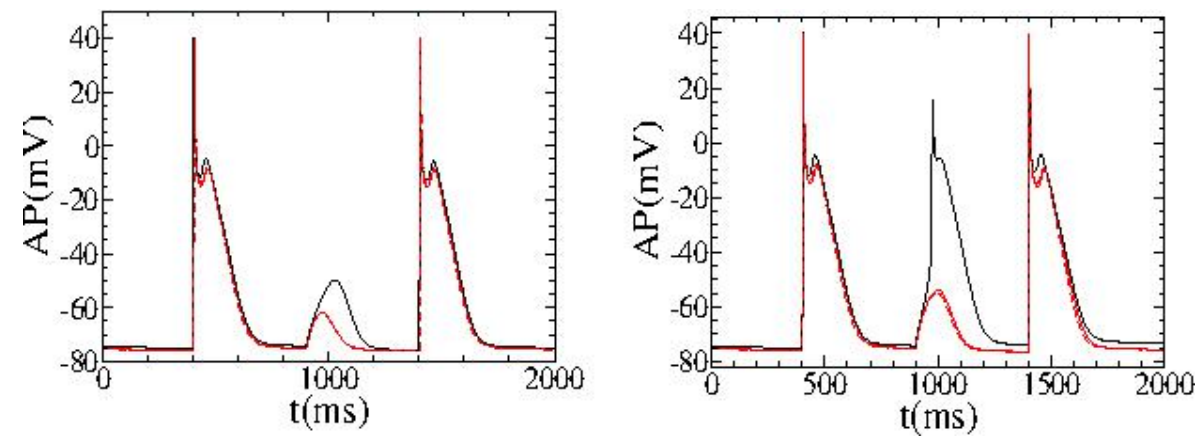

Figure 4. Effect of $I_{\mathrm{KCa}}$ current during a spontaneous calcium release for a stimulation period $T=1000 \mathrm{~ms}$, with $q=2, K_{\mathrm{KCa}}=700 \mathrm{nM}$ and conductance $g=0.005 \mathrm{nS} / \mathrm{pF}$. The percentage of open RyR channels is set to $5 \%$ during $10 \mathrm{~ms}$ (on the left) or to $6 \%$ during $3 \mathrm{~ms}$ (on the right). Red solid lines correspond to the fast gate $\tau=5 \mathrm{~ms}$ and dashed ones to the calcium dependent activation time. Black lines represent the same situation without $I_{\mathrm{KCa}}$.

\section{Discussion and conclusions}

Studies of SK channels have opened an interesting field in cardiac electrophysiology. Controversies about their role in depolarization and arrhythmia are the subject of a wide debate. We have approached this subject by performing simulations of the Lugo model [6], a whole atrial cell model, where the current through these channels has been included. SK channels have been modelled with a sigmoidal calcium dependent gate activation that includes possible variations of cooperativity effects, activation levels and activation time constant dependencies.

Results on the influence of different parameters show a big dependency of the APD with the conductance and gate dynamics. Changes in calcium sensitivity affect the level of the current and the time interval of its effectivity.

Particularly interesting is the appearance of alternans at high conductances for a fast gate when pacing at $500 \mathrm{~ms}$, that together with its effect on APD reduction could explain the pro-arrhythmic effect in the case of channel overexpression.

In contrast, it could be assigned an antiarrhythmic character in the case of spontaneous calcium release, reducing the depolarization of the membrane and avoiding extra depolarizations. In this case it is not clear whether this effect is a consequence of the interrelation of the $I_{\mathrm{KCa}}$ current with the $\mathrm{NaCa}$ exchanger of by the possible variation of the refractory period due to the change in resting potential.

\section{Acknowledgements}

We thank L. Hove-Madsen for fruitful discussions. The authors acknowledge financial support from Fundació La Marató de TV3 and from the Spanish Ministerio de Economía y Competitividad (MINECO) under grant number SAF2014-58286-C2-2-R and FIS2015-66503-C3-2P.

\section{References}

[1] Ellinor PT, Lunetta KL, Glazer NL, et al. Common variants in KCNN3 are associated with lone atrial fibrillation. Nat Genet. 2010; 42:240-244.

[2] Zhang XD, Timofeyev V, Li N, Myers RE, Zhang DM, et al. Critical roles of a small conductance $\mathrm{Ca}$-activated $\mathrm{K}$ channel (SK3) in the repolarization process of atrial myocytes. Cardiovasc Res. 2014; 101:317-325.

[3] Li N, Timofeyev V, Tuteja D, Xu D et al. Ablation of a $\mathrm{Ca}^{2+-}$ activated $\mathrm{k}+$ channel (sk2 channel) results in action potential prolongation in atrial myocytes and atrial fibrillation. The Journal of physiology 2009; 587:10871100.

[4] Hsueh CH, Chang PCh, Hsieh YCh, Reher T, Chen PS, Lin SF. Proarrhythmic effect of blocking the small conductance calcium activated potassium channel in isolated canine left atrium. Heart Rhythm 2013; 10:891898.

[5] Xu Y, Tuteja D, Zhang Z, et al. Molecular identification and functional roles of a $\mathrm{Ca}$-activated $\mathrm{K}$ channel in human and mouse hearts. J Biol Chem 2003: 278:49085-49094.

[6] Lugo CA, Cantalapiedra IR, Peñaranda A, Hove-Madsen L, Echebarria B. Are SR Ca content fluctuations or SR refractoriness the key to atrial cardiac alternans? : insights from a human atrial model. American Journal of Physiology-Heart and Circulatory Physiology 2014; 306: H1540-H1552.

[7] Peñaranda A, Cantalapiedra IR, Alvarez-Lacalle E, Echebarrría B. Electrophysiological effects of small conductance $\mathrm{Ca}^{2+}$-activated channels in atrial myocytes. In: SeMa SiMai Springer Series. Nonlinear dynamics in Biological Systems. In press.

[8] Kennedy M, Bers DM, Chiamvimonvat N, SatoN. Dynamical effects of calcium-sensitive potassium currents on voltage and calcium alternans. The Journal of Physiology 2017; 595: 2285-2297.

Address for correspondence.

Name. Angelina Peñaranda

Full postal address.: Avda Dr Marañón 4408028 Barcelona (Spain)

E-mail address (optional). angelina.penaranda@upc.edu 